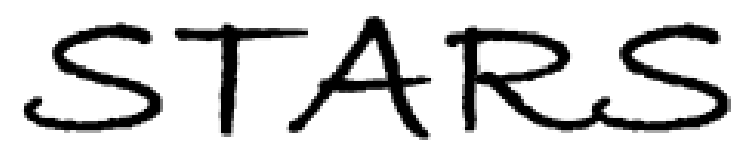

University of Central Florida

STARS

$1-1-2012$

\title{
RKKY interactions in graphene: Dependence on disorder and Fermi energy
}

Hyunyong Lee

E. R. Mucciolo

University of Central Florida

Georges Bouzerar

S. Kettemann

Find similar works at: https://stars.library.ucf.edu/facultybib2010

University of Central Florida Libraries http://library.ucf.edu

This Article is brought to you for free and open access by the Faculty Bibliography at STARS. It has been accepted for inclusion in Faculty Bibliography 2010 s by an authorized administrator of STARS. For more information, please contactSTARS@ucf.edu.

\section{Recommended Citation}

Lee, Hyunyong; Mucciolo, E. R.; Bouzerar, Georges; and Kettemann, S., "RKKY interactions in graphene: Dependence on disorder and Fermi energy" (2012). Faculty Bibliography 2010s. 2917.

https://stars.library.ucf.edu/facultybib2010/2917

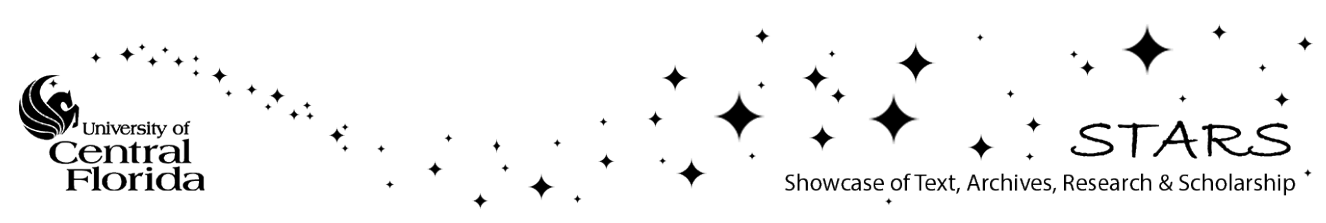




\title{
RKKY interactions in graphene: Dependence on disorder and Fermi energy
}

\author{
Hyunyong Lee, ${ }^{1}$ E. R. Mucciolo, ${ }^{2}$ Georges Bouzerar, ${ }^{3,4}$ and S. Kettemann ${ }^{1,4}$ \\ ${ }^{1}$ Division of Advanced Materials Science, Pohang University of Science and Technology (POSTECH), Pohang 790-784, South Korea \\ ${ }^{2}$ Department of Physics, University of Central Florida, Orlando, Florida 32816, USA \\ ${ }^{3}$ Institut Néel, CNRS, départment MCBT, 25 avenue des Martyrs, BP 166, 38042 Grenoble Cedex 09, France \\ ${ }^{4}$ School of Engineering and Science, Jacobs University Bremen, Bremen 28759, Germany
}

(Received 23 April 2012; revised manuscript received 4 November 2012; published 26 November 2012)

\begin{abstract}
We report, how the indirect exchange interaction $J_{\mathrm{RKKY}}(R)$ between magnetic moments at a distance $R$ in graphene depends on nonmagmetic disorder strength $W$ and gate voltage. First, a semiclassical method is used to rederive $J_{\mathrm{RKKY}}$ in clean graphene, yielding the asymptotic decay $1 / R^{2+\alpha}$, where $\alpha=1$ is the power of the pseudogap at the Dirac point. Next, we perform numerical calculations with the Anderson tight-binding model on a honeycomb lattice. We observe that along the armchair direction $J_{\mathrm{RKKY}}$ is more robust to nonmagnetic disorder than in other directions. This is explained semiclassically by the presence of more than one shortest path between two lattice sites in armchair directions, which is shown to reduce the disorder sensitivity compared to other directions. The distribution of $J_{\mathrm{RKKY}}$ is calculated. We identify three different distribution shapes, repeated periodically along the zigzag direction, while only one kind, more narrow distribution, is observed along the armchair direction. We explain this by the different sensitivity to scattering phases. When increasing $W$, we find that the distribution crosses over to a logarithm-normal distribution. Its width is found to increase linearly with $W$. Moving away from the Dirac point, Friedel oscillations appear in addition to the one caused by the interference between two Dirac points. This results in a beating pattern. We study how this is effected by nonmagnetic disorder.
\end{abstract}

DOI: 10.1103/PhysRevB.86.205427

PACS number(s): $71.70 . \mathrm{Gm}$

\section{INTRODUCTION}

Large.12pt efforts have been devoted to study the electronic transport properties of graphene due to the unusual nature of quasiparticles in this material, which are massless chiral Dirac fermions. A recent experiment, indicating that vacancies in graphene may induce local magnetic moments $(\mathrm{MMs})^{1}$ renewed the interest in its magnetic properties. It is believed that the carrier-mediated effective exchange coupling between MMs, Ruderman-Kittel-Kasuya-Yoshida (RKKY) interaction, plays a crucial role in magnetic properties of graphene. Local probing is possible, using spin-polarized scanning tunneling spectroscopy. A direct measurement of $J_{\text {RKKY }}$ in a conventional metal has been done by measuring the magnetization curves of individual magnetic atoms adsorbed on a platinum surface. ${ }^{2}$ Similar experiments may soon be possible on graphene.

Analytical and numerical studies of $J_{\mathrm{RKKY}}$ in clean graphene at the neutrality point have been reported. ${ }^{3-6} \mathrm{~A}$ dominant feature of graphene is the bipartite nature of its honeycomb lattice. Due to particle-hole symmetry of bipartite lattices, $J_{\text {RKKY }}$ always induces ferromagnetic correlation between MMss on the same sublattice, but antiferromagnetic correlation between MMs on different sublattices. ${ }^{3}$ At the neutrality point, the dependence of the RKKY interaction on the distance $R$ between two MMs in graphene is found to be $1 / R^{3}$, in contrast to the standard behavior in conventional two-dimensional metallic systems where $1 / R^{2}$ is expected. ${ }^{7}$ In doped graphene, but not too far from the neutrality point, the behavior changes to $1 / R^{2}$ and two different length scales control $J_{\mathrm{RKKY}}$ : the wavelength corresponding to the inverse of the distance between the two Dirac points in momentum space, $\left|\boldsymbol{K}-\boldsymbol{K}^{\prime}\right|^{-1}$, and the Fermi wavelength $k_{F}^{-1} \cdot{ }^{6}$ Here, we only consider the static RKKY interaction; for the dynamic case, the decay of the interaction can be slower. ${ }^{7}$
Since the RKKY interaction is mediated by itinerant electrons in the host metal, nonmagnetic defects influence directly these interactions. On-site potential fluctuations scatter the phase of the electron's wave function, altering any interference phenomenon observed in the clean system. The effects of weak nonmagnetic disorder on the exchange interactions in conventional metals have been carefully studied. ${ }^{8-15}$ Numerical work in the strongly localized regime has been done in one-dimensional disordered chains. ${ }^{16}$ These studies found that in the diffusive regime, $J_{\mathrm{RKKY}}$, when averaged over disorder configurations, decays exponentially over distances exceeding the mean free path scale $l_{e}$. However, its geometrical average has the same amplitude as in the clean limit. Only in the localized regime, on length scales $R \gg \xi$ exceeding the localization length $\xi$, its geometrical average is exponentially suppressed, so that $J_{\mathrm{RKKY}}$ is cutoff over distances exceeding $\xi$. In our previous work, we have studied the effect of nonmagnetic disorder on $J_{\mathrm{RKKY}}$ in undoped graphene using the kernel polynomial method (KPM). ${ }^{14,15}$ The unexpected result was that at weak disorder, $J_{\mathrm{RKKY}}$ along the armchair direction is not influenced by nonmagnetic disorder as much as in other directions. Motivated by this unexplained behavior, in this paper, we start by employing the semiclassical method to evaluate $J_{\mathrm{RKKY}}$ in graphene. This calculation helps us to understand the $R$ dependence and the origin of the direction dependence of the sensitivity to disorder seen in Ref. 14. We then use the numerical KPM to calculate $J_{\mathrm{RKKY}}$ in graphene in order to study the effect of disorder and of doping in more detail.

In order to study the disordered conduction electrons in graphene numerically, we employ the single-band Anderson tight-binding model on a honeycomb lattice:

$$
\hat{H}=-t \sum_{\langle i, j\rangle} c_{i}^{\dagger} c_{j}+\sum_{i}\left(w_{i}-\mu\right) c_{i}^{\dagger} c_{i},
$$


where $t$ is the hopping amplitude, $c_{i}$ is an annihilation operator of an electron at site $i, c_{i}^{\dagger}$ is the creation operator, $w_{i}$ is the uncorrelated on-site disorder potential, distributed uniformly between $[-W / 2, W / 2] .\langle i, j\rangle$ indicates nearest-neighbor sites. Periodic boundary conditions are used. Lattice constant $a$ and $\hbar$ are set to unity.

The RKKY exchange coupling is given by

$$
J_{\mathrm{RKKY}}=\frac{-S(S+1)}{4 \pi S^{2}} \int d \omega f(\omega) \Im\left\{G^{\mathrm{r}}\left(\boldsymbol{r}, \boldsymbol{r}^{\prime}, \omega\right) G^{\mathrm{r}}\left(\boldsymbol{r}^{\prime}, \boldsymbol{r}, \omega\right)\right\},
$$

which we evaluate numerically by KPM. ${ }^{14,15}$ We start with the semiclassical derivation of $J_{\mathrm{RKKY}}$ that helps us to understand the numerical results.

\section{SEMICLASSICAL DERIVATION}

Bergmann interpreted the RKKY oscillation as an interference of the conduction electron's wave function scattered by the magnetic impurity and calculated the interference using the Huygens' principle in a three-dimensional metal. ${ }^{17}$ According to the Huygens' principle in two dimensions, ${ }^{18}$ the amplitude of a wave which propagates from a source at position $\boldsymbol{R}^{\prime}$ decays with distance and gains a phase factor at a position $\boldsymbol{R}$ given by $\Psi\left(\boldsymbol{R}^{\prime}\right) \frac{e^{i \boldsymbol{k} \cdot\left(\boldsymbol{R}-\boldsymbol{R}^{\prime}\right)}}{\sqrt{\left|\boldsymbol{R}-\boldsymbol{R}^{\prime}\right|}}$, where $\Psi\left(\boldsymbol{R}^{\prime}\right)$ is the amplitude of the wave at the source and the extra factor comes from the asymptotic form of the Bessel function $\left(e^{i k r} / \sqrt{r}\right)$. If an electron propagates from $\boldsymbol{R}$ to the origin in graphene [see Fig. 1(a)], the amplitude gets a phase factor

$$
A(0, R)=\frac{1}{\sqrt{R}}\left[e^{i(\boldsymbol{K}+\boldsymbol{q}) \cdot \boldsymbol{R}}+e^{i\left(\boldsymbol{K}^{\prime}+\boldsymbol{q}\right) \cdot \boldsymbol{R}}\right] e^{i \varepsilon_{q} t / 2},
$$

where the wave vector is expanded around the two neighboring Dirac points $\boldsymbol{K}$ and $\boldsymbol{K}^{\prime}$ with a relative wave vector $\boldsymbol{q}$. During its propagation, the electron gets another phase factor, $e^{i \varepsilon_{q} t / 2}$, where $\varepsilon_{q}=v_{F} q$ is the kinetic energy of the electron near the Dirac point, $v_{F}$ is the Fermi velocity and $t / 2$ is the propagation time from $\boldsymbol{R}$ to the origin. After the electron is scattered by a magnetic impurity at the origin, it travels back to the position $\boldsymbol{R}$. The amplitude then gains an additional modulation given by

$$
A(R, 0)=\frac{\sin \delta_{0}}{\sqrt{R}}\left[e^{-i(\boldsymbol{K}+\boldsymbol{q}) \cdot \boldsymbol{R}}+e^{-i\left(\boldsymbol{K}^{\prime}+\boldsymbol{q}\right) \cdot \boldsymbol{R}}\right] e^{i\left(\varepsilon_{q} t / 2+\delta_{0}\right)},
$$

(a)

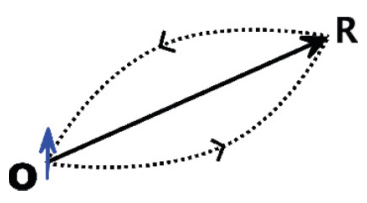

(b)

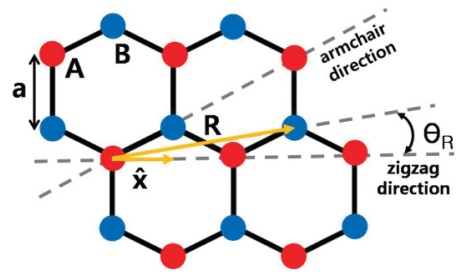

FIG. 1. (Color online) (a) Propagating paths of an electron between positions 0 and $\boldsymbol{R}$. (b) Graphene lattice, where the two sublattices are denoted as $\mathrm{A}$ and $\mathrm{B}$. Two representative directions (zigzag and armchair) are indicated as dashed gray lines. $\theta_{\boldsymbol{R}}$ is the angle between $\boldsymbol{R}$ and the unit vector $\hat{\boldsymbol{x}}$. where $\delta_{0}$ denotes the phase shift due to exchange interaction $J$ of the electron spin with the magnetic impurity spin. ${ }^{17,19}$ When the electron goes back to $\boldsymbol{R}$ its direction is opposite. It yields the opposite sign in the phase of $A(R, 0)$ than $A(0, R)$. It propagates, however, still in the same time direction so that the phase, related to time evolution, $\varepsilon_{q} t / 2$, has the same sign. This is consistent with the diagrammatic expression for $J_{\mathrm{RKKY}}$, which has two retarded Green's functions. For a closed path, a loop of opposite direction makes a contribution with the same weight, so that the modulation of the charge density of an electron with energy $\varepsilon_{q}$ is

$$
\begin{aligned}
\Delta \rho_{\varepsilon}(\boldsymbol{R}) & =|\psi(\boldsymbol{R})|^{2} A(0, R) A(R, 0)+\text { c.c. } \\
& =\frac{2 \sin \left(2 \delta_{0}\right)}{V} \frac{1+\cos \left[\left(\boldsymbol{K}-\boldsymbol{K}^{\prime}\right) \cdot \boldsymbol{R}\right]}{R} e^{i \varepsilon_{q} t},
\end{aligned}
$$

such that the total charge modulation is given by

$$
\begin{aligned}
\rho(\boldsymbol{R}) & =\int d \varepsilon f(\varepsilon) N(\varepsilon) \Delta \rho_{\varepsilon}(\boldsymbol{R}) \\
& =-\frac{\sin \left(2 \delta_{0}\right) v_{F}^{2}}{V} \frac{1+\cos \left[\left(\boldsymbol{K}-\boldsymbol{K}^{\prime}\right) \cdot \boldsymbol{R}\right]}{R^{d+\alpha}} .
\end{aligned}
$$

Here, $|\psi(\boldsymbol{R})|^{2}=1 / V$ for free electrons in a system with volume $V . f(\varepsilon)$ is the Fermi-Dirac distribution function, $N(\varepsilon)=|\varepsilon|^{\alpha}$ the density of states at Fermi energy. $\alpha$ is the pseudogap exponent, $d$ the spatial dimension, and $t=2 r / v_{F}$ the total time it takes to return to $\boldsymbol{R}$.

The total charge density in Eq. (6) can be easily related to $J_{\mathrm{RKKY}} \cdot{ }^{17}$ Since in graphene $d=2$ and $\alpha=1$, the resulting decay is $1 / R^{3}$ consistent with previous works. ${ }^{3-5}$ We conclude that the existence of the pseudogap at the Dirac point causes the faster spatial decay of $J_{\mathrm{RKKY}}$ in graphene than in conventional two-dimensional system $d=2, \alpha=0$. Even though Eq. (6) does not yield an exact expression of $J_{\mathrm{RKKY}}$, and does not yield the correct sublattice dependence of its sign, its amplitude and oscillation period agrees in the asymptotic limit with previous results as obtained by the Green's function method, Eqs. (19) and (26) in Ref. 5. We conclude that the asymptotic limit of $J_{\mathrm{RKKY}}$ in graphene, for distances $R$ such that $\left(\boldsymbol{K}-\boldsymbol{K}^{\prime}\right) \cdot \boldsymbol{R} \gg 1$, can be derived semiclassically. We need to caution that there are further limitations to this semiclassical approach: ${ }^{20}$ as Kane and Mele have shown, ${ }^{21}$ spin-orbit interaction may open a gap $\Delta$ at the Dirac point. It can be easily shown with the diagrammatic expression that $J_{\mathrm{RKKY}}$ then decays exponentially for length scales exceeding $L_{\Delta}=v / \Delta$. However, the semiclassical approach would rather give an oscillatory factor with period $\sim L_{\Delta}$. The reason is that in the simplified semiclassical approach outlined above, the time $t$ is simply substituted by $R / v$, while in the full diagrammatic expression there is a time integral with which one recovers the exponential decay. Thus the applicability of the semiclassical method is limited to $1 /\left(\boldsymbol{K}-\boldsymbol{K}^{\prime}\right) \ll \boldsymbol{R} \ll L_{\Delta}$.

\section{DISORDERED GRAPHENE AT THE DIRAC POINT}

\section{A. Numerical results}

We investigate the effect of on-site nonmagnetic disorder at the Dirac point $\mu=0, W \neq 0$. For each value of $W, 1600$ different disorder configurations were generated and Eq. (2) evaluated by the KPM with a Chebyshev polynomial cutoff 

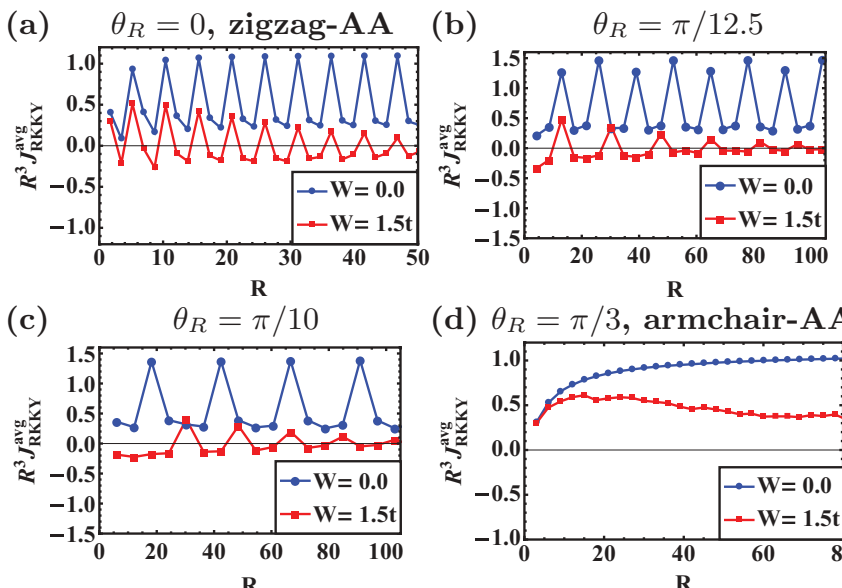

(d) $\theta_{R}=\pi / 3$, armchair-AA

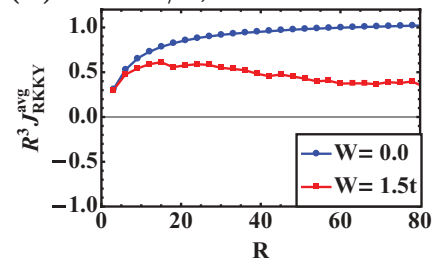

FIG. 2. (Color online) $J_{\text {RKKY }}$ multiplied by $R^{3}$, along (a) $\theta_{R}=0$ (zigzag-AA), (b) $\theta_{R}=\pi / 12.5$, (c) $\theta_{R}=\pi / 10$, and (d) $\theta_{R}=\pi / 3$ (armchair-AA) direction in the diffusive regime, averaged over 1600 disorder configurations. $\theta_{R}$ is defined in Fig. 1(b) $\left(5 \times 10^{5}\right.$ sites, $\left.M=5 \times 10^{3}\right)$.

number $M=5 \times 10^{3}$ on a lattice with $5 \times 10^{5}$ sites. As previously reported, in the diffusive regime $l_{e}<R<\xi$, with mean free path $l_{e}$ and localization length $\xi$, the armchair direction is the only direction in which the disorder averaged $J_{\text {RKKY }}$ does not alter its sign. ${ }^{14}$ To illustrate this, we calculated $J_{\mathrm{RKKY}}$ along the directions $\theta_{R}=\pi / 12.5, \pi / 10$ which pass through only one sublattice. The results are shown in Fig. 2 together with the ones for the zigzag-AA and armchair-AA directions. Notice that $J_{\mathrm{RKKY}}$ is multiplied by $R^{3}$ in order to make the effect of nonmagnetic disorder more transparent. As expected, ${ }^{8-13}$ the average amplitude decays exponentially over length scales exceeding the mean free path $l_{e},\left\langle J_{\mathrm{RKKY}}\right\rangle_{\mathrm{avg}}=$ $J_{\mathrm{RKKY}}^{\text {clean }} e^{-R / l_{e}}$, where $\langle O\rangle_{\text {avg }}$ indicates averaging over disorder configurations and $J_{\mathrm{RKKY}}^{\text {clean }}$ is the RKKY interaction in clean graphene. The mean-free path and localization length in graphene has been extracted from the exponential decay of the average $J_{\mathrm{RKKY}}$ and the typical $J_{\mathrm{RKKY}}$ in our previous study (see Fig. 4 in Ref. 14). On-site disorder breaks the sublattice symmetry. Consequently, the sign of $J_{\text {RKKY }}$ between the MMs which are localized on the same sublattice fluctuates, allowing both ferromagnetic and antiferomagnetic correlation, Figs. 2(a)-2(c). Interestingly, the averaged $J_{\text {RKKY }}$ along the armchair direction with on-site disorder does not change sign, see Fig. 2(d), while for a particular disorder configuration it randomly changes both sign and amplitude. The importance of the sublattice symmetry can be seen by considering the hopping disorder: ${ }^{14}$ when randomness is added to the hopping integral $t=t_{0}+\Delta t$ without on-site disorder, it does not break the sublattice symmetry and $J_{\mathrm{RKKY}}$ never changes its sign.

\section{B. Semiclassical derivation}

In a dirty metal, the charge density is modified by disorder due to the random scattering phases. When these random phase factors are simply averaged over disorder configurations, they give an exponentially decaying factor. Due to the geometrical anisotropy in graphene, the influence of these random phase factors depends on the path direction. According to Feynman's path integral representation, the coupling is dominated by (a) zigzag

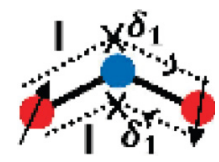

(b) armchair

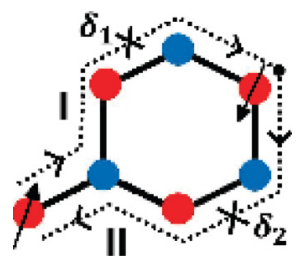

FIG. 3. (Color online) Schematic diagram of the shortest path in (a) the zigzag-AA and (b) armchair-AA directions. $\delta_{1(2)}$ denote the phase shifts due to nonmagnetic disorder.

the shortest path between two MMs. There always exists an even number of shortest paths along the armchair direction, Fig. 3(b), while there is only one along the zigzag direction Fig. 3(a). Therefore, in the zigzag direction, the electron wave is scattered by the same disorder twice with the same phase, $\delta_{1}$ in Fig. 3(a), when it returns to its origin. If we average the modulation of the charge density over the random phase shift $\delta_{1}$, we find

$$
\begin{aligned}
\left\langle\Delta \rho^{\mathrm{zg}}(R)\right\rangle & =\Delta \rho_{0}^{\mathrm{zg}}\left[A_{\mathrm{I}}(0, R) A_{\mathrm{I}}(R, 0)+\text { c.c. }\right] \\
& =\frac{\Delta \rho_{0}^{\mathrm{zg}}}{2}\left\langle e^{i 2 \delta_{1}}+e^{-i 2 \delta_{1}}\right\rangle=\Delta \rho_{0}^{\mathrm{zg}} e^{-2\left\langle\delta_{1}^{2}\right\rangle},
\end{aligned}
$$

where $\langle\cdots\rangle$ is the average over disorder configurations, the identity $\left\langle e^{i \delta}\right\rangle=e^{-\frac{1}{2}\langle\delta\rangle}$ is used and $\Delta \rho_{0}^{\mathrm{zg}}$ is the modulation of charge density along the zigzag direction in the clean system, Eq. (6). However, along the armchair direction, there are closed paths that include different impurities and thereby different scattering phases, $\delta_{1}$ and $\delta_{2}$ in Fig. 3(b). The average of the modulation of charge density is

$$
\begin{aligned}
\langle\Delta & \left.\rho^{\operatorname{arm}}(R)\right\rangle \\
= & \Delta \rho_{0}^{\operatorname{arm}}\left[A_{\mathrm{I}}(0, R) A_{\mathrm{I}}(R, 0)+A_{\mathrm{II}}(0, R) A_{\mathrm{II}}(R, 0)\right. \\
& \left.+A_{\mathrm{I}}(0, R) A_{\mathrm{II}}(R, 0)+A_{\mathrm{II}}(0, R) A_{\mathrm{I}}(R, 0)\right] \\
= & \Delta \rho_{0}^{\operatorname{arm}} \frac{1}{4}\left\langle e^{i 2 \delta_{1}}+e^{i\left(\delta_{1}+\delta_{2}\right)}+e^{-i\left(\delta_{1}+\delta_{2}\right)}+e^{i 2 \delta_{2}}\right\rangle \\
= & \Delta \rho_{0}^{\operatorname{arm} \frac{1}{2}}\left[e^{-2\left\langle\delta_{1}^{2}\right\rangle}+e^{-\left\langle\delta_{1}^{2}\right\rangle}\right],
\end{aligned}
$$

where we used $\left\langle\delta_{1}\right\rangle=\left\langle\delta_{2}\right\rangle=0$ and $\left\langle\delta_{1}^{2}\right\rangle=\left\langle\delta_{2}^{2}\right\rangle$.

The modulation along the armchair direction Eq. (8) is dominated by the term $\Delta \rho_{0} e^{-\left\langle\delta_{1}^{2}\right\rangle}$ coming from the closed loop connected by two unrelated paths, so that the average value is exponentially closer to the clean value, while the zigzag direction is dominated by $\Delta \rho_{0} e^{-2\left\langle\delta_{1}^{2}\right\rangle}$, Eq. (7). Thus one may expect $J_{\mathrm{RKKY}}$ in the armchair direction to be less sensitive to the nonmagnetic weak disorder than in other directions. This may explain our previous results ${ }^{14}$ and the results shown in Fig. 2, where the averaged value in the armchair direction remains substantially larger than in the other directions and does not change its sign.

\section{Distribution of RKKY interaction}

In order to investigate in more detail how nonmagnetic disorder affects $J_{\text {RKKY }}$ and depends on the direction in the graphene lattice and on particular lattice points, we have calculated the distribution of its amplitude for a disorder strength $W=t$ at sites on the zigzag and armchair direction. The results are shown in Figs. 4(a)-4(e). When the distance 

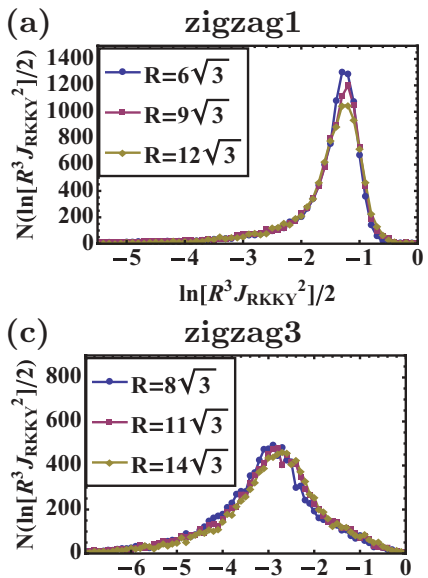

$\ln \left[R^{3} J_{\mathrm{RKKY}}{ }^{2}\right] / 2$

(e)

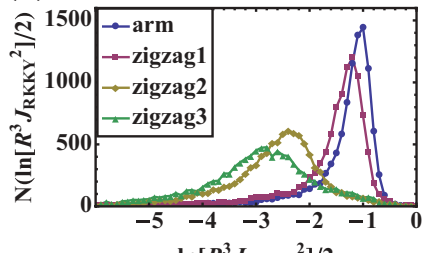

$\ln \left[R^{3} J_{\mathrm{RKKY}}{ }^{2}\right] / 2$

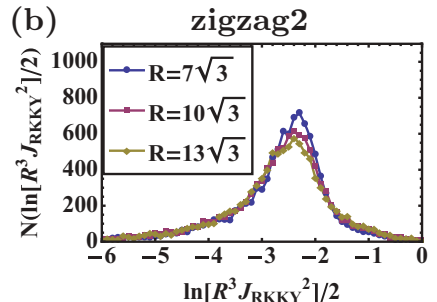

(d)

armchair

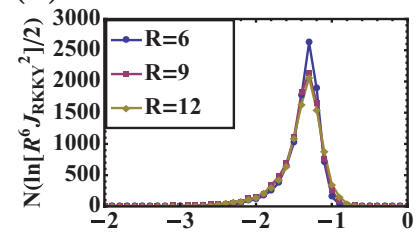

$\ln \left[R^{6} J_{\mathrm{RKKY}}{ }^{2}\right] / 2$

(f)



FIG. 4. (Color online) The distribution of $\sqrt{J_{\text {RKKY }}^{2}}$ multiplied by $R^{3}$, for (a) the first, (b) second, (c) third of the triplet of points along zigzag-AA direction, see Fig. 4(f), and (d) for the armchair direction. For comparison, these distributions are plotted together in (e) ( $W=t$, $2 \times 10^{4}$ sites $M=10^{3}$ ).

$R$ between the MMs is smaller than the mean-free path $l_{e}$, $J_{\text {RKKY }}$ multiplied by $R^{3}$ has a shape, which is independent on $R$. Interestingly, the distribution has three different shapes along the zigzag-AA direction, see Figs. 4(a)-4(c), which are repeated periodically every third site in that direction. However, there is only one type of distribution along the armchair direction, as can be seen in Fig. 4(d). For direct comparison, we plot all distributions together in Fig. 4(e). This remarkable difference can be explained in the following way: the argument of the oscillating factor in the semiclassical expression for the charge density, $\left(\boldsymbol{K}-\boldsymbol{K}^{\prime}\right) \cdot \boldsymbol{R}$, Eq. (6), takes only the values, $\left(0, \frac{2 \pi}{3}, \frac{4 \pi}{3}\right)$, modulo $2 \pi$, along sites in the zigzag-AA direction. $J_{\mathrm{RKKY}}$ at sites where the argument of the oscillating factor takes the values $\left(\frac{2 \pi}{3}, \frac{4 \pi}{3}\right)$ along the zigzag-AA direction has the broadest (green) distribution, while at sites where the argument takes the value $(0)$, modulo $2 \pi$, it has the narrowest (red) distribution. Indeed, the disorder is expected to shift the oscillation for a given disordered sample by some phase $\delta$ as $\cos \left[\left(\boldsymbol{K}-\boldsymbol{K}^{\prime}\right) \cdot \boldsymbol{R}+\delta\right]$. If we expand that oscillating factor in $\delta$ for sites where $\left(\boldsymbol{K}-\boldsymbol{K}^{\prime}\right) \cdot \boldsymbol{R}=0$, modulo $2 \pi$, the leading order correction is proportional to $\delta^{2}$. If we expand it however, for the sites where $\left(\boldsymbol{K}-\boldsymbol{K}^{\prime}\right) \cdot \boldsymbol{R}=\left(\frac{2 \pi}{3}, \frac{4 \pi}{3}\right)$, the first correction is linear in $\delta$. This explains why $J_{\mathrm{RKKY}}$ is indeed more sensitive to disorder at such sites, and should have a wider distribution, in agreement with Fig. 4. The unaveraged $J_{\text {RKKY }}$ in a particular disorder representation is found to remain long ranged as in the clean system. However, we find that the oscillations acquire a random phase that also changes with the distance $R$ between magnetic impurities. Therefore the average value does not characterize $J_{\mathrm{RKKY}}$ at
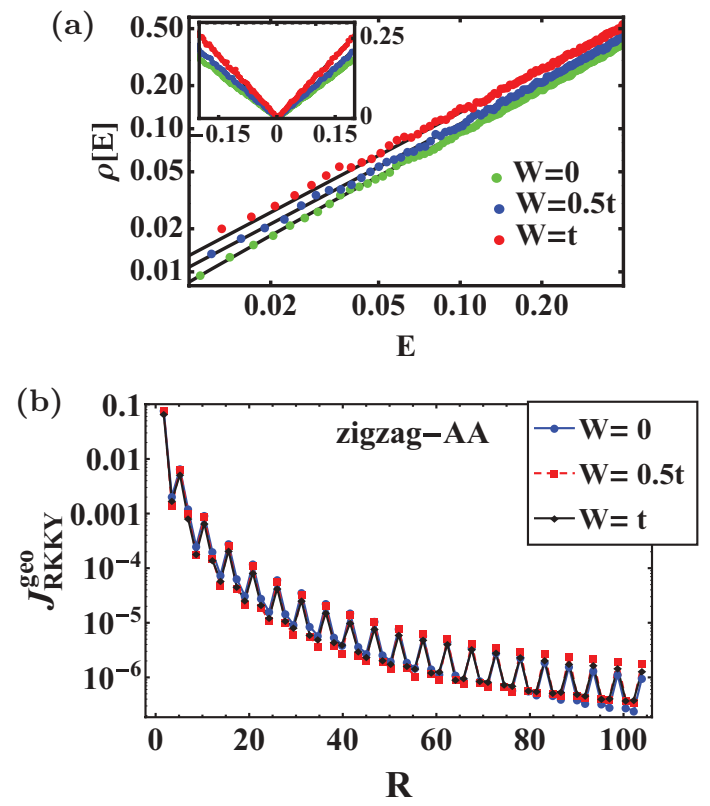

FIG. 5. (Color online) (a) Averaged density of states in a double logarithmic plot. Inset: linear plot $\left(M=7 \times 10^{3}\right)$. (b) Geometrical averaged $J_{\text {RKKY }}$ along zigzag-AA direction $\left(M=5 \times 10^{3}\right) .3 \times 10^{7}$ sites are used. Black lines in (a) are fittings to $\rho(E)=\gamma|E|$ with $\gamma=0.95,1.07,1.3$ for $W=0,0.5 t, t$, respectively.

distances exceeding the mean free path. To find the typical value, we evaluated the geometrical average of the interaction amplitude, defined by $J_{\text {RKKY }}^{\text {geo }} \equiv \exp \left[\frac{1}{2}\left\langle\ln \left(J_{\text {RKKY }}\right)^{2}\right\rangle_{\text {avg }}\right]$. This typical value is found to have the same power-law decay with $R$ as in the clean limit. ${ }^{11-13,16,17}$ We have calculated the density of states for two disorder strengths $W=0.5 t, t$ to observe how weak disorder affects the pseudogap at the neutrality point $(E=0)$, since, as we have seen above, the power of the pseudogap is directly related to the unconventional power-law decay of $J_{\text {RKKY }}$. We used the KPM ${ }^{22,23}$ method. As we see in Fig. 5(a), the pseudogap is stable at weak disorder, and has the same power law as in the clean limit. Only its slope around the Dirac point is changed ${ }^{23} \rho(E)=\gamma|E|$, where $\rho(E)$ is the density of states and $E$ is the energy measured from the Dirac point in units of the hopping amplitude $t$. The slope $\gamma$ depends on the disorder strength as obtained by fitting the data in Fig. 6. An analytical study has reported that there is a logarithmic correction to the density of states around the neutrality point, yielding $\rho(E)=|E| \ln |E|$ in the presence of disorder. ${ }^{24}$ This logarithmic correction does not change the power of the distance dependence of the RKKY interaction Eq. (6). Consequently, the geometrical average of $J_{\mathrm{RKKY}}$ is expected to have the same power-law exponent as the clean system $1 / R^{3}$. The direct numerical calculation shown in Fig. 5(b) strongly supports this conclusion.

Figure 6(a) shows how $J_{\text {RKKY }}$ in a strongly disordered sample gets suppressed by several orders of magnitude when $W$ is increased. In order to investigate the broadening of the distribution, we employed $3 \times 10^{4}$ realizations of the disorder potential and calculated $J_{\text {RKKY }}$ for a fixed distance $R=50 \sqrt{3}$. The results are shown in Fig. 6(b). The inset indicates that it has a distribution with very long asymmetric tails. The squared amplitude $J_{\mathrm{RKKY}}^{2}$ has a distribution similar 

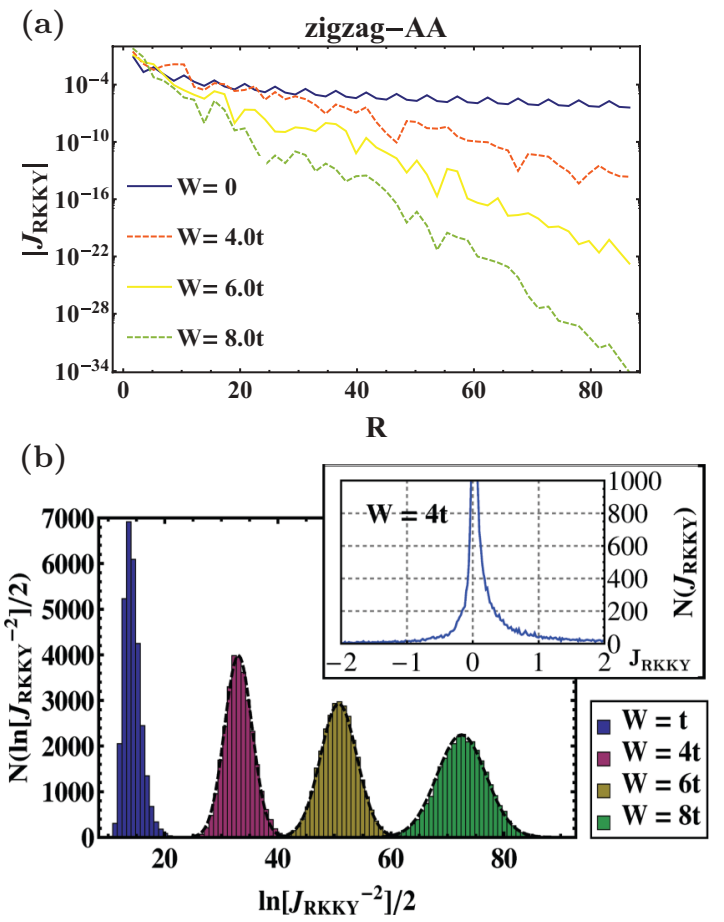

FIG. 6. (Color online) (a) Absolute value of $J_{\mathrm{RKKY}}$ in a disordered sample as a function of distance $R$. (b) The distribution of the logarithm of $\left|J_{\text {RKKY }}\right|$ for $R=50 \sqrt{3} a$ and different $W$. The inset of (b) shows the distribution of the amplitude $J_{\mathrm{RKKY}}$ itself for $W=4 t$. $1.8 \times 10^{5}$ sites and $M=3 \times 10^{3}$ are used. Black dashed lines are logarithm-normal distribution functions with $\sigma=2.4,3.2,4.35$ and $\alpha=32.5,50,72.5$ for $W=4 t, 6 t, 8 t$, respectively (see text).

to log-normal with a width that increases with disorder strength. Using a field-theoretical approach valid in the metallic regime, Lerner found ${ }^{12}$ that the increase in the strength of the nonmagnetic disorder leads to a crossover in the shape of the distribution function from a broad non-Gaussian with the very long tails in the weak disorder regime to a completely logarithm-normal distribution in the region of strong disorder regime. This may explain the crossover of the distribution that we observe from weak disorder $W=t$ to strong disorder $W=4 t, 6 t, 8 t$. For direct comparison, we fitted the results with a logarithm-normal function given by $P(x)=\frac{N}{\sqrt{2 \pi \sigma^{2}}} \exp \left[-\frac{(x-\alpha)^{2}}{2 \sigma^{2}}\right]$, where $N=3 \times 10^{4}$ is the number of realizations and $x=\ln \left[J_{\mathrm{RKKY}}^{-2}\right] / 2$. The data are shown together with the fitting curves in Fig. 6(b) (black dashed lines). We analyzed the width $\sigma$ of the distribution Eq. (IIIC) as a function of the disorder strength $W$ and is shown in Fig. 7. The red line in Fig. 7, $\sigma=W / 2+0.34$, agrees qualitatively with the analytical prediction by Lerner, who has used the renormalization group method to obtain ${ }^{12}$ $\sigma \sim 1 / \sqrt{l_{e}} \sim W$, where $l_{e} \propto 1 / W^{2}$ is the mean free path of electrons, which we studied in Ref. 14.

\section{DOPED GRAPHENE $(\mu \neq 0)$}

A. Clean $\operatorname{system}(\boldsymbol{W}=\mathbf{0})$

By varying the Fermi level $\mu$ in the Hamiltonian of Eq. (1), we investigate how $J_{\text {RKKY }}$ evolves with $\mu$. Results are shown in

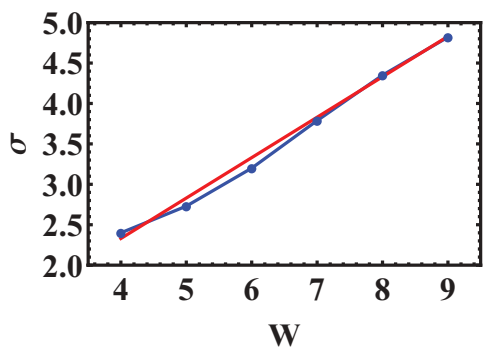

FIG. 7. (Color online) Plot of the width of the distribution of $\left|J_{\mathrm{RKKY}}\right|$ as a function of $W$ in units of $t$. Red line: linear fitting, yielding $\sigma=W / 2+0.34$.

Fig. 8, where $J_{\text {RKKY }}$ is multiplied by $R^{2}$ in order to emphasize its oscillatory behavior. In these calculations, we used a lattice with $7.2 \times 10^{5}$ sites and $M=3 \times 10^{3}$. Near the Dirac point a beating pattern appears as shown in Figs. 8(a) and 8(b). It consists of a superposition of waves with the wave vectors $\boldsymbol{K}-\boldsymbol{K}^{\prime}$ and $\boldsymbol{q}_{F}$, where $\boldsymbol{q}_{F}$ is the Fermi wave vector originating from the Friedel oscillations at finite Fermi energy. Recently, the following analytical expressions for the beating pattern were derived using lattice Green's functions: ${ }^{5}$

$$
J_{\mathrm{AB}}=J_{\mathrm{AB}}^{0}\left[1-\frac{8 q_{F} R}{\sqrt{3 \pi}} G_{2,4}^{2,1}\left(\begin{array}{c}
\frac{1}{2}, \frac{3}{2} \\
1,2,0,-\frac{1}{2}
\end{array} ; q_{F}^{2} R^{2}\right)\right],
$$

where $J_{\mathrm{AB}}^{0}$ is the RKKY coupling function at the neutrality point and $G$ is the Meijer- $G$ function. Note that term within brackets describes the isotropic dependence of the oscillations

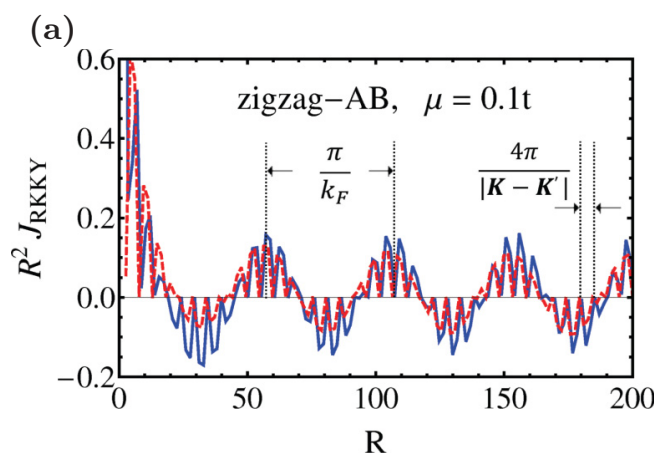

(b)

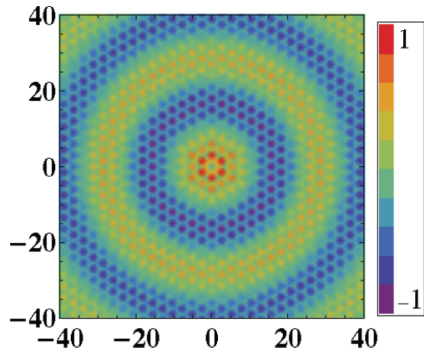

(c)

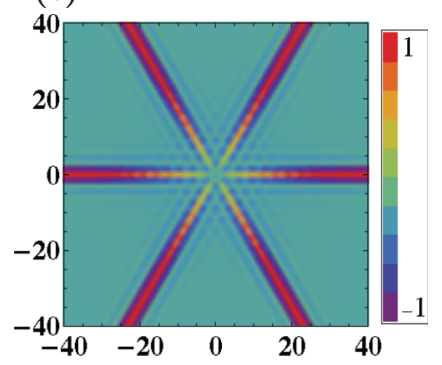

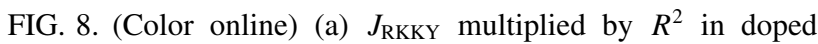
graphene at the chemical potential $\mu=0.1 t$ along the zigzag-AB direction. Density plots of $J_{\mathrm{RKKY}}$ for (b) $\mu=0.2 t$ and (c) $\mu=t$ for all directions. In (a), the results of calculations with KPM and the lattice Green's function method are shown as solid blue and dashed red lines, respectively. $\left(7.2 \times 10^{5}\right.$ sites, $M=5 \times 10^{3}$.) 
(a)

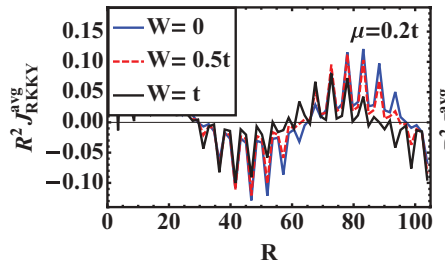

(b)

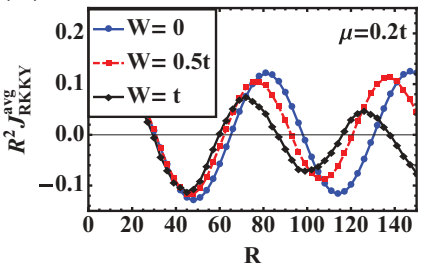

FIG. 9. (Color online) Average $J_{\mathrm{RKKY}}$ multiplied by $R^{2}$, along (a) zigzag-AA, (b) armchair-AA directions at $\mu=0.2 t$ in the diffusive regime. $N=1600$. Lattice size and $M$ as in Fig. 2.

on the Fermi momentum $\boldsymbol{q}_{F}$. The external prefactor $J_{\mathrm{AA}(\mathrm{B})}^{0}$ on the contrary, is strongly anisotropic, depending on the vector given by the momentum difference between the two neighbored Dirac points $\boldsymbol{K}-\boldsymbol{K}^{\prime}$. To make a comparison with our calculations, the function represented by Eq. (9) is also plotted in Fig. 8, red dashed line. Excellent agreement is found. One can estimate the wavelength of the long oscillation, which appears at finite $\mu$, using the dispersion relation near the neutrality point given by $E(\boldsymbol{q})=v_{F}|\boldsymbol{q}|$, where $\boldsymbol{q}$ is the momentum relative to the Dirac point and $v_{F}=3 \mathrm{ta} / 2$ is the Fermi velocity. ${ }^{25}$ The Fermi wavelength is found to be about $\lambda_{F} \approx 50 a$ for $\mu=0.1 t$. This coincides with the period seen in Fig. 8(a). As expected from Eq. (9), the oscillations with large period seen in [see Fig. 8(b)] are isotropic.

We have also calculated $J_{\mathrm{RKKY}}$ for highly doped graphene $(\mu=t)$. The results are multiplied by the square of the distance, $R^{2}$, and are shown in the density plots of Fig. 8(c). The behavior cannot be described by Eq. (9), which is valid only close to the Dirac point. When $\mu$ is exactly at the van Hove singularity $\mu=t$, the ordering pattern of $J_{\mathrm{RKKY}}$ along the zigzag direction is reversed. In other words, the correlation between MMs on zigzag-AA or BB is always antiferromagnetic and ferromagnetic for zigzag- $\mathrm{AB}$ or $\mathrm{BA}$ pairs. At the same time, $J_{\mathrm{RKKY}}$ is strongly suppressed for other directions. This is in accordance with a result of a previous study. ${ }^{26}$

\section{B. Disordered system}

We have also calculated the $J_{\mathrm{RKKY}}$ in disordered graphene at finite $\mu$. The results are shown in Fig. 9. A lattice with $5 \times 10^{5}$ sites and $M=5 \times 10^{3}$ were used. Even though the unusual oscillation coming from the interference of two Dirac points still exists Fig. 9(a), the amplitude of the envelope of the averaged $J_{\text {RKKY }}$ decreases with disorder strength $W$ and the period $2 \pi / k_{\mathrm{F}}$ of the oscillation coming from the finite $\mu$ is modified by the disorder as in a conventional metal.

\section{CONCLUSIONS}

In conclusion, we have studied the RKKY interaction $J_{\mathrm{RKKY}}$ in graphene as a function of disorder strength $W$ and chemical potential $\mu$ in detail, by using the kernel polynomial method. We also employed a semiclassical analysis of $J_{\mathrm{RKKY}}$, deriving the modulation of the charge density in the presence of MMs. This provides an intuitive interpretation of the origin of the oscillating behavior of $J_{\mathrm{RKKY}}$ as the interference of the two degenerate Dirac points. With this semiclassical approach we could also trace the origin of the unusual power-law decay of $J_{\mathrm{RKKY}}$ in pure graphene at the Dirac point directly to the pseudogap in the density of states.

In order to study the anisotropic influence of nonmagnetic disorder, we evaluated $J_{\mathrm{RKKY}}$ along two different directions between the zigzag and armchair directions. As reported in our previous study, in the diffusive regime, the armchair direction is not affected by the nonmagnetic disorder as much as the other directions. Using the semiclassical approach, we could trace that anisotropic sensitivity of $J_{\mathrm{RKKY}}$ to disorder to the presence of multiple shortest paths between two MMs in the armchair direction, showing that this direction is more robust to disorder than other directions. We also found that in the ballistic regime $R<l_{e}$, the distribution of $J_{\mathrm{RKKY}}$ along the zigzag direction is not universal but depends on the lattice sites at which the pair of magnetic impurities sit. We identified three different representative shapes, which repeat themselves periodically. We found a semiclassical explanation of this effect. By an accurate evaluation of the density of states around the Dirac point in weakly disordered regime $W \leqslant t$ we confirmed that the pseudogap is not filled by weak, uncorrelated disorder. This is in full agreement with the fact that the geometrical average of $J_{\mathrm{RKKY}}$ in the diffusive regime decays as in the clean system at the Dirac point, namely as $1 / R^{3}$, and confirms that this anomalous behavior is related to the power of the pseudogap, $r=1$. In the localized regime $R>\xi$, the geometrical average is exponentially suppressed at distances exceeding the localization length $\xi$ and the distribution of the absolute value of $J_{\mathrm{RKKY}}$ shows a crossover from a non-Gaussian shape with very long tails to the logarithm-normal form when increasing $W$. We analyzed its width and confirmed that it increases linearly with the amplitude of the disorder potential $W$.

Finite gate voltage breaks particle-hole symmetry and the resulting finite Fermi surface yields Friedel oscillations, so that the sign of $J_{\mathrm{RKKY}}$ between MMs localized on the same sublattice now oscillates with distance. When the Fermi level is exactly at the van Hove singularity $\mu=t$, the ordering pattern of $J_{\mathrm{RKKY}}$ along the zigzag direction is reversed. At the same time, the interactions are strongly suppressed for all other directions. The disorder sensitivity of $J_{\mathrm{RKKY}}$ becomes at finite gate voltage isotropic as in a conventional disordered metal.

In this work, we showed that the KPM method is efficient and accurate for studying interactions in disordered systems. In order to minimize the computation time while keeping the highest accuracy, the convergence of the calculations with respect to the Chebyshev polynomial cutoff degree $M$ and the system size $L$ have been investigated in detail (see Appendix). The proper cutoff $M$ to reach convergence is found to increase linearly with distance $R$ between two MMs, as seen in Fig. 10(b). A system size $L$ about five times larger than $R$ is found to give convergent results. In comparison with the exact numerical diagonalization, the KPM is found to be much faster. It can be implemented for very large system sizes in disordered systems, where thousands of realizations are needed in order to yield meaningful statistics. KPM is thereby established as an efficient tool to study magnetic properties of disordered systems. 
(a)

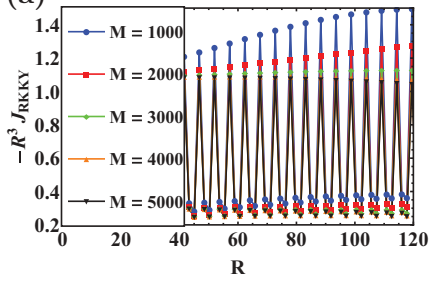

(b)

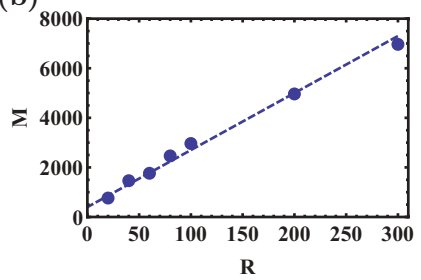

FIG. 10. (Color online) (a) $J_{\text {RKKY }}$ as a function of $M\left(5 \times 10^{5}\right.$ sites). (b) The smallest $M$ that yields a convergent result as a function of $R$.

\section{ACKNOWLEDGMENTS}

This research was supported by WCU (World Class University) program through the National Research Foundation of Korea funded by the Ministry of Education, Science and Technology(Grant No. R31-2008-000-10059-0), Division of Advanced Materials Science. E.R.M. acknowledges partial support through the NSF DMR (Grant No. 1006230). E.R.M. and G.B. thank the WCU AMS for its hospitality. H. Y. Lee thanks Jacobs University Bremen for its hospitality.

\section{APPENDIX: CONVERGENCE OF THE KERNEL POLYNOMIAL METHOD CALCULATIONS}

When using the KPM the calculation of the Chebyshev polynomials using recurrence relations consumes most of the computation time. Therefore we investigated first the relation between the cutoff number $M$ and the convergence of the results in order to be able to minimize $M$ and optimize the calculations. For clean graphene, a lattice with $5 \times 10^{5}$ sites

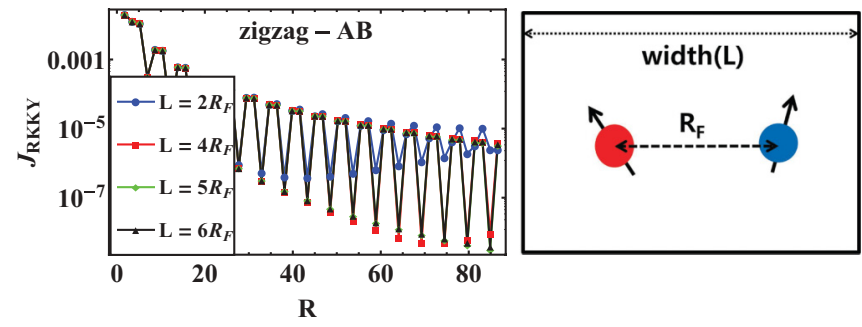

FIG. 11. (Color online) $J_{\mathrm{RKKY}}$ as a function of system size $L$. $50 \sqrt{3} a$ is used as the longest distance $R_{F}\left(M=5 \times 10^{3}\right)$.

was used in these calculations. When a cutoff number $M$ is not sufficient, the amplitude of $J_{\text {RKKY }}$ deviates from the expected power-law behavior, as indicated by the blue and red lines in Fig. 10(a). When we determined the smallest cutoff number $M$ such that the variance of the amplitude of $J_{\mathrm{RKKY}}$ is less than $5 \%$, we found that it increases linearly with the distance between two MMs $R$ as seen in Fig. 10(b). This linear relation between the distance and the cutoff number allows the rapid calculation of $J_{\mathrm{RKKY}}(R)$.

In order to minimize the KPM calculation time further, we have also studied the smallest system size $L$ which yields convergent results, as shown in Fig. 11. $R_{F}$ denotes the longest distance used in the calculation, $R_{F}=50 \sqrt{3} a$, the cutoff number is $M=5 \times 10^{3}$. One can observe a good convergent behavior (see green and black lines in Fig. 11) when system size $L$ is larger than $5 R_{F}$. The exact diagonalization method also yields a proper result when $L=5 R_{F} .{ }^{4}$ However, the KPM does not require matrix diagonalization and therefore is much faster.
${ }^{1}$ J.-H. Chen, L. Li, W. G. Cullen, E. D. Williams, and M. S. Fuhrer, Nat. Phys. 7, 1745 (2007).

${ }^{2}$ F. Meier, L. Zhou, J. Wiebe, and R. Wiesendanger, Science 320, 82 (2008).

${ }^{3}$ S. Saremi, Phys. Rev. B 76, 184430 (2007).

${ }^{4}$ A. M. Black-Schaffer, Phys. Rev. B 81, 205416 (2010).

${ }^{5}$ M. Sherafati and S. Satpathy, Phys. Rev. B 83, 165425 (2011).

${ }^{6}$ M. Sherafati and S. Satpathy, Phys. Rev. B 84, 125416 (2011).

${ }^{7}$ S. R. Power, F. S. M. Guimarães, A. T. Costa, R. B. Muniz, and

M. S. Ferreira, Phys. Rev. B 85, 195411 (2012).

${ }^{8}$ P. G. De Gennes, J. Phys. Radium 23, 630 (1962).

${ }^{9}$ P. F. de Chatel, J. Magn. Mater. 23, 28 (1981).

${ }^{10}$ A. Jagannathan, E. Abrahams, and M. J. Stephen, Phys. Rev. B 37, 436 (1988).

${ }^{11}$ A. I. Zyuzin and B. Z. Spivak, JEPT Lett. 243, 43 (1986).

${ }^{12}$ I. V. Lerner, Phys. Rev. B 48, 9462 (1993).

${ }^{13}$ L. N. Bulaevskii and S. V. Panyukov, JETP Lett. 43, 240 (1986).

${ }^{14}$ H. Lee, J. Kim, E. R. Mucciolo, G. Bouzerar, and S. Kettemann, Phys. Rev. B 85, 075420 (2012).

${ }^{15}$ H. Lee, E. R. Mucciolo, G. Bouzerar, and S. Kettemann, Int. J. Mod. Phys.: Conf. Ser. 11, 177 (2012).
${ }^{16}$ J. A. Sobota, D. Tanasković, and V. Dobrosavljević, Phys. Rev. B 76, 245106 (2007)

${ }^{17}$ G. Bergmann, Phys. Rev. B 36, 2469 (1987).

${ }^{18}$ M. A. Cirone, J. P. Dahl, M. Fedorov, D. Greenberger, and W. P. Schleich, J. Phys. B 35, 191 (2002).

${ }^{19}$ A. C. Hewson, The Kondo Problem to Heavy Fermions, Cambridge Studies in Magnetism (Cambridge University Press, Cambridge, 1993).

${ }^{20}$ We would like to thank a referee for raising this question.

${ }^{21}$ C. L. Kane and E. J. Mele, Phys. Rev. Lett. 95, 226801 (2005).

${ }^{22}$ A. Weiße, G. Wellein, A. Alvermann, and H. Fehske, Rev. Mod. Phys. 78, 275 (2006).

${ }^{23}$ M. Amini, S. A. Jafari, and F. Shahbazi, Europhys. Lett. 87, 37002 (2009).

${ }^{24}$ B. Y.-K. Hu, E. H. Hwang, and S. Das Sarma, Phys. Rev. B 78, 165411 (2008)

${ }^{25}$ A. H. Castro Neto, F. Guinea, N. M. R. Peres, K. S. Novoselov, and A. K. Geim, Rev. Mod. Phys. 81, 109 (2009).

${ }^{26}$ B. Uchoa, T. G. Rappoport, and A. H. Castro Neto, Phys. Rev. Lett. 106, 016801 (2011). 